\title{
Real-Time Cellular Imaging of Protein Poly(ADP-ribos)ylation
}

\author{
Annette Buntz, Sarah Wallrodt ${ }^{+}$,Eva Gwosch, Michael Schmalz, Sascha Beneke, \\ Elisa Ferrando-May, Andreas Marx,* and Andreas Zumbusch*
}

\begin{abstract}
Poly(ADP ribos)ylation (PARylation) is an impor tant posttranslational protein modification, and is involved in major cellular processes such as gene regulation and DNA repair. Its dysregulation has been linked to several diseases, including cancer. Despite its importance, methods to observe PARylation dynamics within cells are rare. By following a chemical biology approach, we developed a fluorescent $\mathrm{NAD}^{+}$analogue that proved to be a competitive building block for protein PARylation in vitro and in cells. This allowed us to directly monitor the turnover of PAR in living cells at DNA damage sites after near infrared (NIR) microirradiation. Addi tionally, covalent and noncovalent interactions of selected target proteins with PAR chains were visualized in cells by using FLIM FRET microscopy. Our results open up new opportunities for the study of protein PARylation in real time and in live cells, and will thus contribute to a better under standing of its significance in a cellular context.
\end{abstract}

ver the last decade, biochemical investigations and in vivo studies have revealed the importance of poly(ADP ribos)yl ation (PARylation, Figure $1 \mathrm{~A}$ ) as a complex, functionally diverse posttranslational modification. ${ }^{[1]}$ Protein modification through covalent attachment of ADP ribose is mediated by a family of enzymes called ADP ribosyl transferases with diphtheria toxin homology (ARTDs), which comprises 17 members in humans. By using nicotinamide adenine dinucle otide $\left(\mathrm{NAD}^{+}\right)$as a substrate, ARTDs transfer ADP ribose covalently onto glutamate, aspartate, or lysine residues of acceptor proteins. Mono(ADP ribos)ylated proteins are formed through the release of nicotinamide. Among several ARTDs which are able to elongate this initial ADP ribose to PAR polymers, ${ }^{[2]}$ ADP ribosyl transferase with diphtheria

[*] A. Buntz, ${ }^{[+]}$Prof. Dr. A. Zumbusch

Department of Chemistry and Center for Applied Photonics

University of Konstanz

Universitätsstraße 10, 78457 Konstanz (Germany)

E mail: andreas.zumbusch@uni konstanz.de

S. Wallrodt, ${ }^{[+]}$Prof. Dr. A. Marx

Department of Chemistry, University of Konstanz

Universitätsstraße 10, 78457 Konstanz (Germany)

E mail: andreas.marx@uni konstanz.de

E. Gwosch, Dr. S. Beneke, Prof. Dr. E. Ferrando May

Department of Biology, University of Konstanz

Universitätsstraße 10, 78457 Konstanz (Germany)

M. Schmalz

Department of Physics and Center for Applied Photonics

University of Konstanz

Universitätsstraße 10, 78457 Konstanz (Germany)

$\left.{ }^{+}\right]$These authors contributed equally.

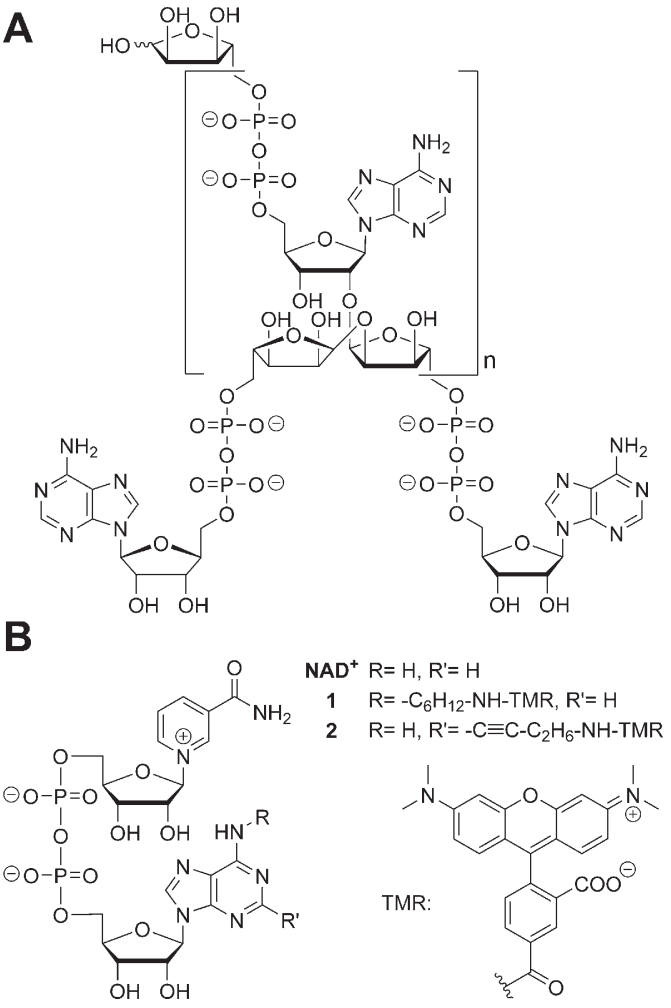

Figure 1. A) Structure of poly (ADP ribose). B) Structures of $\mathrm{NAD}^{+}$and the $\mathrm{NAD}^{+}$analogues $\mathbf{1}$ and $\mathbf{2}$.

toxin homology 1 (ARTD1) is considered to be the main source of PAR. ${ }^{[3]}$ ARTD1 is involved in the detection and repair of DNA damage, transcriptional regulation, and modulation of RNA processing. ${ }^{[4]}$ Dysregulation of ARTD1 function is associated with cancer, inflammatory processes, neurodegeneration, and cardiovascular diseases, which makes it an attractive clinical target. ${ }^{[5]}$

Protein PARylation is a tightly regulated process, which, apart from PAR synthesizing enzymes, also involves degrad ing enzymes such as poly(ADP ribose) glycohydrolases (PARGs). ${ }^{[6]}$ The effects of PARylation on protein function are mediated either by 1) modulation of the binding affinity of protein interaction sites as a result of covalent PAR attachment, 2) disruption of protein complexes, or 3) the recruitment of other proteins. The latter two processes rely on noncovalent binding of proteins to the spatially extended and flexible polymer. The rapid fluctuation of cellular PAR levels makes the molecular study of protein PARylation a demand ing task. To date, only few methods exist for the visualization of PARylation in cells, and none offer the possibility to directly follow the dynamic behavior of PAR in response to stimuli such as induction of DNA damage. On the one hand, 
indirect cellular imaging can be achieved by using PAR antibodies (e.g., $10 \mathrm{H}$ ) or $^{[7]}$ ADP ribose binding macrodo mains (e.g., of macroH2A), ${ }^{[8]}$ as well as through tagging PAR synthesizing enzymes (e.g., EGFP ARTD1). ${ }^{[9]}$ On the other hand, direct visualization of PAR can be realized by using functionalized $\mathrm{NAD}^{+}$analogues. For this purpose, bioorthog onal reactions based on the use of reporter tagged NADs have been developed for the investigation of ADP ribosyla tion in vitro ${ }^{[10]}$ and in cells. ${ }^{[11]}$ In this case, fluorescent labeling is achieved by reacting the incorporated $\mathrm{NAD}^{+}$analogue with suitably functionalized fluorescent dyes. Although these reactions are relatively fast, they are performed in fixed cells after PAR formation is completed. Hence, despite their potential impact on the study and understanding of PAR mediated protein regulation, ${ }^{[12]}$ methods that enable live cell imaging of PAR formation on the polymer level do not exist so far. Herein, we report on the development of fluorescently labeled $\mathrm{NAD}^{+}$analogues for the time resolved visualization of PARylation in live cells (Figure 1B). Additionally, we show that the application of these $\mathrm{NAD}^{+}$analogues in combination with fluorescence lifetime imaging (FLIM) enables the protein specific detection and study of PARylation in cells after exposure to genotoxic damage.

$\mathrm{NAD}^{+}$probes have successfully been applied in ADP ribosylation reactions using 2 and 6 modified purine ana logues. ${ }^{[10,11]}$ We therefore synthesized $\mathrm{NAD}^{+}$analogues modified with fluorescent dyes at these positions via suitable linkers. First, we prepared modified adenosine monophos phates bearing amino linkers and reacted them with tetra methylrhodamine (TMR) $N$ hydroxysuccinimide ester (Fig ure $1 \mathrm{~B}$ and Figures S1, S2 in the Supporting Information). Consecutive coupling of the obtained dye modified mono phosphates with pre activated $\beta$ nicotinamide monophos phate resulted in the desired fluorophore labeled NADs $\mathbf{1}$ and 2. $^{[10 \mathrm{~b}, 11 \mathrm{a}]}$

With these compounds in hand, we tested their substrate properties in conventional ADP ribosylation assays with histone H1.2 or ARTD1 itself as acceptors. ${ }^{[10 \mathrm{~b}]}$ To this end, the respective analogues were incubated with the proteins and short dsDNA as an ARTD1 activator. Incorporation of the modified NADs was judged by SDS PAGE analysis, by comparing the fluorescence signals to the Coomassie Blue staining. When the reactions were performed in the absence of natural $\mathrm{NAD}^{+}$, modification of $\mathrm{H} 1.2$ with fluorescent PAR chains was observed only for compound 2 (Figure 2, lane 4 vs. 7). Upon applying a 1:1 mixture with natural NAD ${ }^{+}$, we found that PAR chains were also formed with 1 (Figure 2, lane 8). It is noteworthy that the 2 modified analogue results in shorter PAR chains (Figure 2, lane 5). This suggests that the modification might affect polymer elongation. Interestingly, incubation in the absence of enzyme leads to some minor staining of the histone (see Figure 2, lanes 3 and 6). This might be due to electrostatic attraction between the negatively charged $\mathrm{NAD}^{+}$analogue and the positively charged protein and/or to the non enzymatic addition of ADP ribose at lysine residues through Schiff base formation. ${ }^{[13]}$ Additionally, we tested the $\mathrm{NAD}^{+}$analogues in an auto(ADP ribos)ylation assay (Figure S3), where ARTD1 serves as its own acceptor. $^{[14]}$ Here too, we observed the fluorescent label

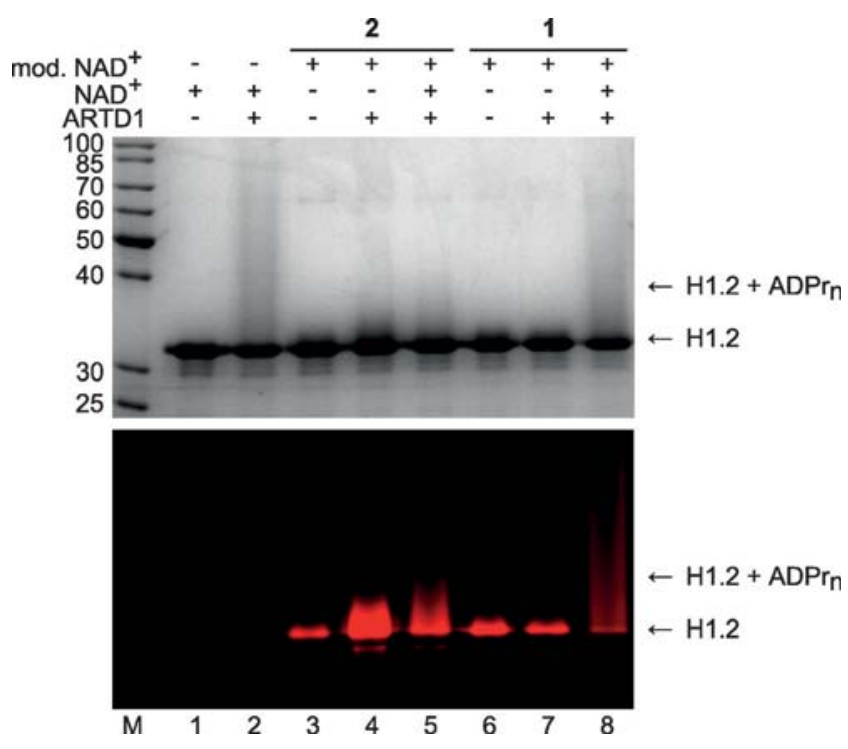

Figure 2. Comparison of $\mathrm{NAD}^{+}$analogues $\mathbf{1}$ and $\mathbf{2}$ in the PARylation of histone $\mathrm{H1}$.2, as resolved by SDS PAGE. Upper panel: Coomassie Blue staining, lower panel: TMR fluorescence signals. The total concentra tion of NADs was $1 \mathrm{~mm}$. Controls were performed using either natural substrate (lanes 1 and 2) or no enzyme (lanes 1, 3, and 6). Each analogue was also tested in a 1:1 ratio with natural NAD $^{+}$(lanes 5 and 8).

indicative of auto modified ARTD1 only when applying 2 (Figure S3, lane 3 vs. 4). Our findings suggest 1 ) that the initial attachment and polymerization of ADP ribose(s) and the subsequent elongation of already existing chains are differ ently affected by the introduced modification, and 2) that 2 better competes against the natural substrate during initiation than 1. Considering that PAR chains formed in cells are significantly shorter than those formed in vitro, ${ }^{[15]}$ a cellular $\mathrm{NAD}^{+}$probe must prove efficient enough in both initiation and elongation. Since these criteria are better met by $\mathbf{2}$, we continued our experiments using this analogue only. This compound was next studied in a PARylation assay of histone H1.2 with different ratios of modified to unmodified NAD ${ }^{+}$ (Figure 3). Our results show that PAR formation can be detected within a wide range of ratios. At ratios of modified $\mathrm{NAD}^{+} /$unmodified NAD ${ }^{+}$below 1:5, almost no differences in polymer formation are visible in vitro (Figure 3, lanes $610 \mathrm{vs.}$ 11). This demonstrates that compound $\mathbf{2}$ is a competitive substrate for protein PARylation.

Based on these findings, we speculated that $\mathbf{2}$ is indeed suited for studying protein PARylation in living cells. The uptake of the negatively charged $\mathrm{NAD}^{+}$across the cell membrane was achieved by using the carrier peptide Pep 1. ${ }^{[16]}$ We first tested different cargo/carrier ratios and analyzed the labeling efficiency by flow cytometry. In addition, cell viability was estimated by Sytox Blue staining under varying conditions. We found that more than $90 \%$ of the cells were efficiently labeled after one hour of incubation with $25 \mu \mathrm{M}$ of analog 2 and $100 \mu \mathrm{M}$ Pep 1. Cell viability was not affected at any of the applied concentrations (Figure S4). Live cell imaging of PAR dynamics at sites of DNA damage was performed at a confocal microscope equipped with a femto 


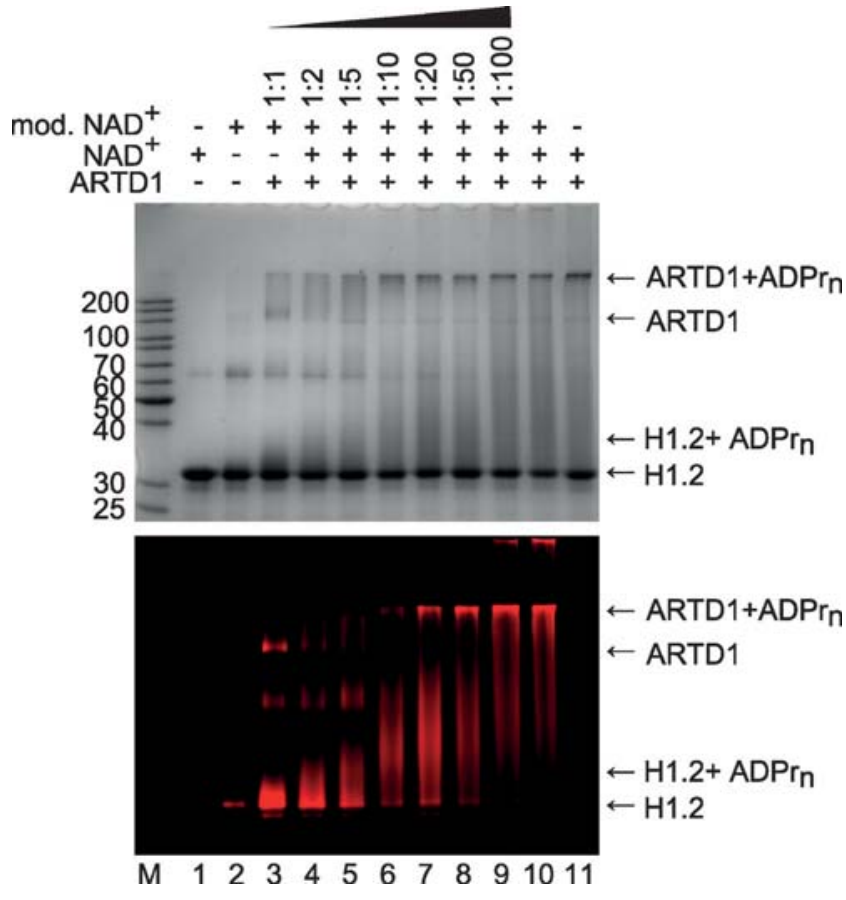

Figure 3. SDS PAGE analysis of the PARylation of histone H1.2 using $\mathrm{NAD}^{+}$analogue 2 and natural $\mathrm{NAD}^{+}$. Upper panel: Coomassie Blue staining, lower panel: TMR fluorescence signals. The total concentra tion of NADs was $1 \mathrm{~mm}$, with different ratios of modified to unmodi fied $\mathrm{NAD}^{+}$as indicated. Controls were performed using either natural substrate (lanes 1 and 11) or no enzyme (lanes 1 and 2).

second fiber laser source. After cellular loading of 2, cell nuclei were microirradiated with femtosecond pulses at a wavelength of $775 \mathrm{~nm}$ along a line track (Figure 4A), thereby resulting in the activation of ARTD1 as previously described ${ }^{[17]}$ DNA damage results from three photon excita tion and is spatially confined in three dimensions to a volume corresponding to the focal spot. ${ }^{[18]}$ We recorded PAR formation in the laser irradiated tracks through confocal fluorescence imaging at a wavelength of $555 \mathrm{~nm}$ to detect the redistribution of $\mathbf{2}$ within the nucleus at intervals of $10 \mathrm{~s}$ (Figure 4B). Image data were quantified by measuring the enhancement of the fluorescence signal in the irradiated region of interest relative to the surrounding area (Fig ure $4 \mathrm{C}$ ). Within seconds after the induction of DNA damage, a strong increase in fluorescence intensity was observed, followed by a slower decay in fluorescence intensity, which ensued after approximately $50 \mathrm{~s}$ (Video S1 in the Supporting Information). As a control, the same experiment was performed in the presence of the ARTD inhibitor ABT888. ${ }^{[19]}$ Under these conditions, redistribution of $\mathbf{2}$ in the microirradiated track was completely abrogated, thus indicating that the accumulation of $\mathbf{2}$ and its processing at the DNA damage site can be attributed to ARTD1 activity.

In order to directly visualize the modification of ARTD1 with fluorescently labeled PAR, we performed an additional series of experiments using FLIM FRET microscopy. This approach has successfully been used for the detection of other posttranslational modifications in a protein specific manner. ${ }^{[20]}$ It is based on the observation of Förster resonance energy transfer (FRET) between two neighboring fluoro phores. We thus fused the FRET donor EGFP to the backbone of ARTD1, while the TMR label present on the $\mathrm{NAD}^{+}$analogue functions as the FRET acceptor (Figure $5 \mathrm{~A}$ ). Upon modification of EGFP ARTD1 with TMR labeled PAR, FRET can be detected through a reduction in the fluorescence lifetime of EGFP, which is one of the most direct and robust readouts of FRET (FLIM FRET microscopy, see Supporting Information for details).

As a first set of experiments, we performed fluorescence lifetime measurements of EGFP ARTD1 in vitro, using either the purified protein (Figure S5) or lysates of HEK293T cells expressing EGFP ARTD1 (Figure 5B). The reaction mixture, containing the $\mathrm{NAD}^{+}$analogue $\mathbf{2}$, natural $\mathrm{NAD}^{+}$, and dsDNA, was incubated at $37^{\circ} \mathrm{C}$ and PARylation was monitored in real time. Consecutive modification of EGFP ARTD1 with multiple TMR labeled ADP ribose units leads to a prominent decrease in the EGFP fluorescence lifetime, which is indicative of increasing FRET. The effect was not observed when the reaction was performed in the absence of dsDNA or upon addition of the ARTD inhibitor olaparib. ${ }^{[21]}$ We confirmed that the EGFP tag did not impair ARTD1 activity by comparing wild type ARTD1 and EGFP ARTD1 in ADP ribosylation assays (Figures S6, S7).
A

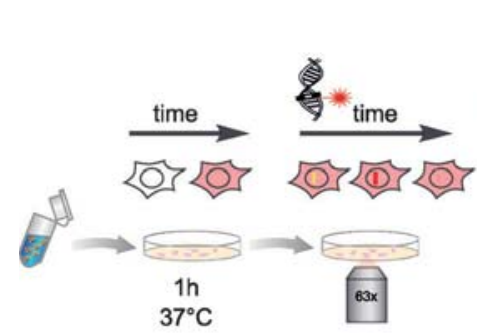

B

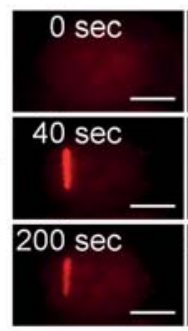

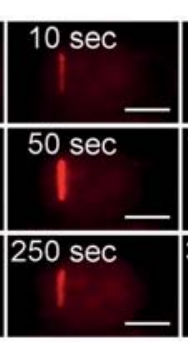

C

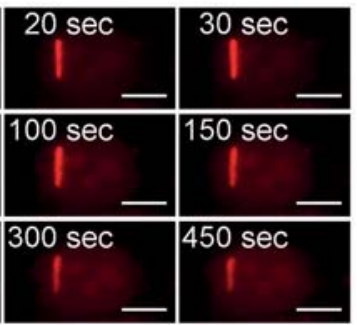

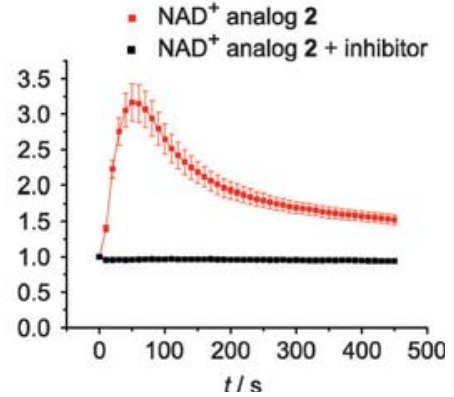

Figure 4. Live cell monitoring of PARylation upon DNA damage by using NAD ${ }^{+}$analogue 2. A) A scheme illustrating the experimental setup. Hela cells were loaded with 2 and microirradiated to induce DNA damage. B) An exemplary confocal microscopy image sequence. Scale bars: 7 m. C) Quantitative analysis of the relative enhancement of the fluorescence signal at DNA damage site compared to the surrounding area ( $n 32$ cells). Control experiments were performed with $10 \mu \mathrm{M}$ ARTD inhibitor ABT888 ( $n \quad 28$ cells). Three independent experiments were performed. Error bars represent standard errors of the mean (SEM). 
A
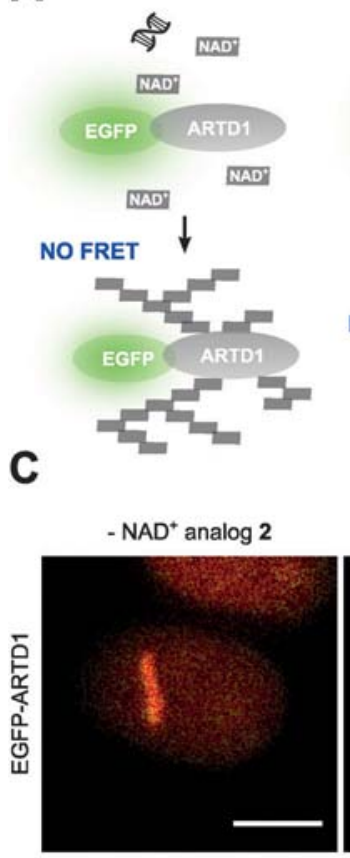

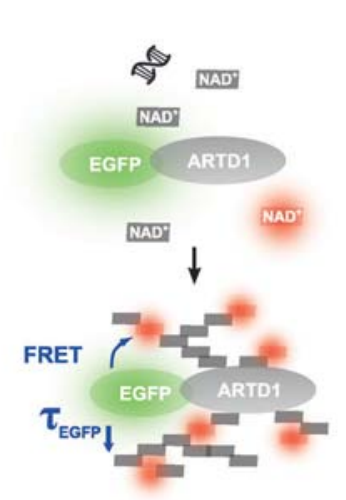

$+\mathrm{NAD}^{+}$analog $2 \quad \tau(\mathrm{ns})$

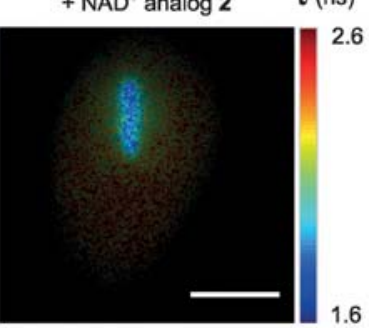

B

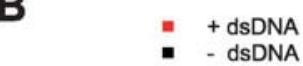

- + dsDNA + ARTD inhibitor

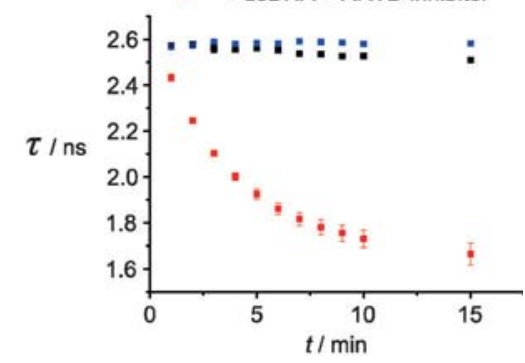

D

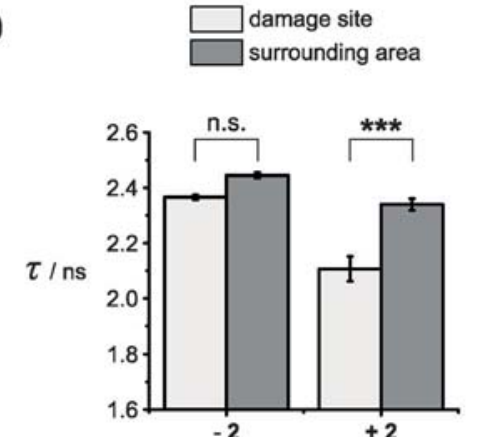

Figure 5. Fluorescence lifetime based detection of PARylation. A) Activation of ARTD1 leads to FRET between EGFP and the TMR label at the PAR polymer, as measured by a decrease in the EGFP fluorescence lifetime. B) HEK293T cells expressing EGFP ARTD1 were lysed and the extracts were incubated with dsDNA, NAD ${ }^{+}$analogue 2 , and natural $\mathrm{NAD}^{+}$. Control experiments were conducted without dsDNA or with the ARTD inhibitor olaparib. Fluorescence lifetimes were measured at $37^{\circ} \mathrm{C}$. Error bars represent the SEM, $n$ 3. C) Hela cells expressing EGFP ARTD1 were treated with 2 or left untreated, microirradiated to induce DNA damage, and fixed. Fluorescence lifetime images of EGFP ARTD1 are intensity weighed and presented in pseudocolors. Scale bars: $7 \mu \mathrm{m}$. D) Quantitative analysis of fluores cence lifetimes from 15 cells. Mean values \pm SEM are depicted. Statistical significance was assessed with a two way ANOVA and Bonferroni posttest. The level of significance is given with n.s. not significant, **** $p<0.001$.

Having successfully employed fluorescence lifetime meas urements in vitro, we aimed to image PAR protein interac tions in cells. To this end, Hela cells expressing EGFP ARTD1 and loaded with $\mathbf{2}$ were subjected to laser micro irradiation as described above. This led to the enrichment of both EGFP and TMR fluorescence signals at the irradiated sites (Figure S8 A). Cells were fixed two to three minutes after irradiation and analyzed on a FLIM microscope (Figure 5C). The fluorescence lifetime of EGFP ARTD1 was measured for every image pixel and displayed by means of intensity weighted pseudocolors (i.e., pixels with higher intensity appear brighter). DNA damage sites are identified by a bright EGFP fluorescence signal, which results from the recruitment of EGFP ARDT1. In control samples without fluorescently labeled $\mathrm{NAD}^{+}$, the EGFP fluorescence lifetime was invariant throughout the nucleus. By contrast, in the presence of 2, regions of high EGFP intensity show a signifi cantly reduced fluorescence lifetime of the donor caused by FRET between EGFP ARTD1 and the TMR labeled PAR. This observation is confirmed by a quantitative analysis of fluorescence lifetimes measured in 15 cells (Figure 5D).

An important mode of action of PARylation is the recruitment of proteins to PAR chains through noncovalent interaction. However, methods that have been employed to identify and characterize spe cific noncovalent PAR binding protein domains ${ }^{[22]}$ cannot be applied in the context of intact cells. Because FRET relies on proximity between two fluores cent labels, we reasoned that our approach is not only suited to following covalent modifica tion with PAR but should also enable the detection of nonco valent interactions of PAR with proteins. We tested this hypoth esis by using a macroH2A EGFP fusion protein as FRET donor. ${ }^{[8 b]}$ This highly conserved protein domain is known to bind ADP ribose in a noncova lent manner. ${ }^{[8]}$ Hela cells expressing macroH2A EGFP were thus treated with $\mathbf{2}$, irradi ated, and subjected to FLIM FRET analysis as described above (Figures S8B,S9A). In the area surrounding the irradi ated track, the macroH $2 \mathrm{~A}$ EGFP fluorescence lifetimes did not differ between TMR treated and untreated cells. Importantly, the EGFP fluores cence lifetime at the DNA damage site was significantly reduced only in cells treated with 2 (Figure S9B and S9C). Therefore, noncovalent interactions between macroH $2 \mathrm{~A}$ EGFP and fluorescently labeled PAR chains can also readily be visualized by FLIM FRET microscopy.

In summary, we have described the potential of a novel fluorescently modified $\mathrm{NAD}^{+}$analogue as a probe for the optical imaging of protein PARylation. For this purpose, the probe was covalently modified with the fluorophore TMR. Despite this bulky modification, the $\mathrm{NAD}^{+}$analogue proved to be a competitive and efficient substrate for ARTD1 in vitro as well as in cells. We achieved live cell imaging of both the formation and degradation of PAR upon photoinduced DNA damage. To the best of our knowledge, this is the first time that PAR turnover has been directly monitored on the polymer level in living cells. In combination with FLIM FRET microscopy, the novel analogue $\mathbf{2}$ is a powerful tool for monitoring protein specific PARylation in cells. Our results thus provide the basis for future experiments where both covalent and noncovalent interactions with PAR are studied for selected proteins. In vitro studies such as enzyme specific screening for novel and clinically relevant ARTD1 inhibitors will greatly benefit from this method, since fluorescence lifetimes provide a fast readout of ARTD1 activity in real time. We envision that our approach will be extremely useful 
in live cell studies of PARylation for the identification and characterization of both different PAR synthesizing and PAR degrading enzymes and hitherto unknown protein targets, which has not so far been possible in the context of intact cells.

\section{Acknowledgements}

This work was supported by the Deutsche Forschungsge meinschaft (SFB969, TP B08) and the Konstanz Research School Chemical Biology. We acknowledge the "Deutsche Telekom Stiftung" (A.B.) and the "Beilstein Institut zur Förderung der Chemischen Wissenschaften" (S.W.) for sti pends and the FlowKon facility of the University of Konstanz for support with flow cytometry analysis. The plasmid pcDNA3.1 macroH2A1.1 GFP was kindly provided by A. Ladurner. We thank A. Leitenstorfer for scientific advice on microirradiation with femtosecond laser pulses.

Keywords: DNA repair . FRET - NAD ${ }^{+}$- poly(ADP ribose) . posttranslational modifications

[1] a) L. Virág, Mol. Aspects Med. 2013, 34, 1043 1045; b) W. L. Kraus, Mol. Cell 2015, 58, 902910.

[2] B. A. Gibson, W. L. Kraus, Nat. Rev. Mol. Cell Biol. 2012, 13, 411424.

[3] J. C. Ame, V. Rolli, V. Schreiber, C. Niedergang, F. Apiou, P. Decker, S. Muller, T. Hoger, J. Menissier de Murcia, G. de Mur cia, J. Biol. Chem. 1999, 274, 1786017868.

[4] K. W. Ryu, D. S. Kim, W. L. Kraus, Chem. Rev. 2015, 115, 2453 2481.

[5] a) P. Bai, C. Cantó, Cell Metab. 2012, 16, 290 295; b) F. Y. Feng, J. S. de Bono, M. A. Rubin, K. E. Knudsen, Mol. Cell 2015, 58 , 925934.

[6] K. Hatakeyama, Y. Nemoto, K. Ueda, O. Hayaishi, J. Biol. Chem. 1986, 261, 1490214911.

[7] J. H. Küpper, L. van Gool, M. Müller, A. Bürkle, Histochem. J. 1996, 28, 391395.

[8] a) G. I. Karras, G. Kustatscher, H. R. Buhecha, M. D. Allen, C. Pugieux, F. Sait, M. Bycroft, A. G. Ladurner, EMBO J. 2005, 24, 1911 1920; b) G. Timinszky, S. Till, P. O. Hassa, M. Hothorn, G. Kustatscher, B. Nijmeijer, J. Colombelli, M. Altmeyer, E. H. K. Stelzer, K. Scheffzek, M. O. Hottiger, A. G. Ladurner, Nat. Struct. Mol. Biol. 2009, 16, 923929.
[9] O. Mortusewicz, J. C. Amé, V. Schreiber, H. Leonhardt, Nucleic Acids Res. 2007, 35, 76657675.

[10] a) H. Jiang, J. H. Kim, K. M. Frizzell, W. L. Kraus, H. Lin, J. Am. Chem. Soc. 2010, 132, 9363 9372; b) Y. Wang, D. Rösner, M. Grzywa, A. Marx, Angew. Chem. Int. Ed. 2014, 53, 8159 8162; Angew. Chem. 2014, 126, 8298 8301; c) I. Carter O'Connell, H. Jin, R. K. Morgan, L. L. David, M. S. Cohen, J. Am. Chem. Soc. 2014, 136, 52015204.

[11] a) S. Wallrodt, A. Buntz, Y. Wang, A. Zumbusch, A. Marx, Angew. Chem. Int. Ed. 2016, 55, 7660 7664; Angew. Chem. 2016, 128, 7790 7794; b) E. Bakondi, P. Bai, É. Szabó, J. Hunyadi, P. Gergely, C. Szabó, L. Virág, J. Histochem. Cytochem. 2002, 50, 9198.

[12] N. P. Westcott, H. C. Hang, Curr. Opin. Chem. Biol. 2014, 23, 56 62.

[13] E. Kun, A. C. Chang, M. L. Sharma, A. M. Ferro, D. Nitecki, Proc. Natl. Acad. Sci. USA 1976, 73, 31313135.

[14] J. P. Gagné, C. Ethier, D. Defoy, S. Bourassa, M. F. Langelier, A. A. Riccio, J. M. Pascal, K. M. Moon, L. J. Foster, Z. Ning, D. Figeys, A. Droit, G. G. Poirier, DNA Repair 2015, 30, 6879.

[15] a) O. Popp, S. Veith, J. Fahrer, V. A. Bohr, A. Bürkle, A. Mangerich, ACS Chem. Biol. 2013, 8, 179 188; b) M. Malanga, S. Bachmann, P. L. Panzeter, B. Zweifel, F. R. Althaus, Anal. Biochem. 1995, 228, 245251.

[16] M. C. Morris, J. Depollier, J. Mery, F. Heitz, G. Divita, Nat. Biotechnol. 2001, 19, 11731176.

[17] J. M. F. Fischer, O. Popp, D. Gebhard, S. Veith, A. Fischbach, S. Beneke, A. Leitenstorfer, J. Bergemann, M. Scheffner, E. Ferrando May, A. Mangerich, A. Bürkle, FEBS J. 2014, 281, 36253641.

[18] D. Trautlein, M. Deibler, A. Leitenstorfer, E. Ferrando May, Nucleic Acids Res. 2010, 38, e14.

[19] C. K. Donawho et al., Clin. Cancer Res. 2007, 13, 27282737.

[20] a) W. Lin, L. Gao, X. Chen, Curr. Opin. Chem. Biol. 2015, 28 , 156 163; b) F. Doll, A. Buntz, A. K. Späte, V. F. Schart, A. Timper, W. Schrimpf, C. R. Hauck, A. Zumbusch, V. Wittmann, Angew. Chem. Int. Ed. 2016, 55, 2262 2266; Angew. Chem. 2016, 128, 23032308.

[21] K. A. Menear, C. Adcock, R. Boulter, X. 1. Cockcroft, L. Copsey, A. Cranston, K. J. Dillon, J. Drzewiecki, S. Garman, S. Gomez, H. Javaid, F. Kerrigan, C. Knights, A. Lau, V. M. Loh, I. T. W. Matthews, S. Moore, M. J. O'Connor, G. C. M. Smith, N. M. B. Martin, J. Med. Chem. 2008, 51, 65816591.

[22] J. Krietsch, M. Rouleau, E. Pic, C. Ethier, T. M. Dawson, V. L. Dawson, J. Y. Masson, G. G. Poirier, J. P. Gagne, Mol. Aspects Med. 2013, 34, 10661087. 